\title{
Hyperprogression after immunotherapy in patients with malignant tumors of digestive system
}

\author{
Zhi Ji ${ }^{\dagger}$, Zhi Peng ${ }^{\dagger}$, Jifang Gong, Xiaotian Zhang, Jian Li, Ming Lu, Zhihao Lu and Lin Shen ${ }^{*}$ (1)
}

\begin{abstract}
Background: Immune checkpoint inhibitors (ICls) were approved to have a significant antitumor activity in various tumor types. In practice, some patients do not seem to benefit from ICls but rather to have accelerating disease. The aim of this study was to evaluate hyperprogression in patients with malignant tumors of digestive system treated with ICls.

Methods: Medical records from consecutive patients with malignant tumors of digestive system treated with ICls in Peking University Cancer Hospital were retrospectively collected. Tumor growth kinetics (TGK) on immunotherapy and TGK preimmunotherapy were collected and TGK ratio (TGKR) was calculated. Hyperprogression was defined as TGKR $\geq 2$.

Results: From August 2016 to May 2017, 25 evaluable patients were identified from 45 patients with malignant tumors of digestive system. Five patients were considered as having hyperprogression. Three of 5 were neuroendocrine carcinomas (NECs) and the other 2 were adenocarcinomas. Four of 5 were treated with programmed cell death ligand 1 (PD-L1) inhibitor, the other one was treated with PD-L1 inhibitor combined with cytotoxic T lymphocyte associated antigen-4 (CTLA-4) inhibitor. Pseudoprogression was observed in 2 patients.

Conclusions: Hyperprogression was observed in a fraction of patients with malignant tumors of digestive system treated with ICls. Further investigation is urgently needed.
\end{abstract}

Keywords: Hyperprogression, Immunotherapy, Digestive system, Tumor growth kinetics (TGK), irRECIST

\section{Background}

Immunotherapy has become a new method to refractory or recurrent tumors. A number of clinical studies have confirmed that immune checkpoint inhibitors (ICIs) had a significant antitumor activity in various tumor types [1-5]. The new immunotherapy also results in novel tumor response patterns such as delayed tumor response or pseudoprogression [6, 7]. What's more, researchers found that ICIs might have a deleterious effect by accelerating the disease in a subset of patients which was described as "hyperprogressive disease" or "hyperprogression" [8-10].

Champiat et al. [8] reported occurrences of rapid progression on ICIs and described as "hyperprogressive disease" for the first time. Hyperprogression was defined as a RECIST progression at the first evaluation and as $\mathrm{a} \geq$

\footnotetext{
* Correspondence: shenlin@bjmu.edu.cn

${ }^{\dagger}$ Zhi Ji and Zhi Peng contributed equally to this work

Department of Gastrointestinal Oncology, Key Laboratory of Carcinogenesis and Translational Research (Ministry of Education), Peking University Cancer Hospital \& Institute, Fucheng Road 52, Haidian District, Beijing 100142, China
}

2 -fold increase of the tumor growth rate (TGR) compared with pre-immunotherapy. Nine percent $(12 / 131)$ of evaluable patients were considered as having hyperprogression. Kato et al. [9] observed same phenomenon and attempted to explore the genetic markers associated with hyperprogression. Time to treatment failure (TTF) $<2$ months, $>50 \%$ increase in tumor burden and $>2$-fold increase in progression pace (PP) were considered as hyperprogression. Saada-Bouzid et al. [10] investigated hyperprogression in recurrent and/or metastatic head and neck squamous cell carcinoma (R/M HNSCC) patients. Hyperprogression was defined as $\geq 2$-fold increase of the tumor growth kinetics (TGK) compared with preimmunotherapy. Hyperprogression was observed in $29 \%$ $(10 / 34)$ patients.

We also recently identified a subset of patients with malignant tumors of digestive system whose disease paradoxically accelerated on immunotherapy. Herein, we describe 
our patients of hyperprogressors and discuss the related questions to hyperprogression.

\section{Methods \\ Patients}

Medical records from consecutive patients with malignant tumors of digestive system enrolled and treated in phase I clinical trials with programmed cell death-1/ programmed cell death ligand 1 (PD-1/PD-L1) inhibitor alone or combined with cytotoxic $\mathrm{T}$ lymphocyte associated antigen-4 (CTLA-4) inhibitor in Peking University Cancer Hospital between August 2016 and May 2017 were retrospectively collected (NCT02825940, NCT02978482, NCT02915432, NCT03167853, CTR20160872). All patients had histologically confirmed malignant tumors of digestive system.

\section{Assessments}

$\mathrm{T}_{\mathrm{PRE}}, \mathrm{T}_{0}$, and $\mathrm{T}_{\mathrm{POST}}$ stand for the time of pre-baseline, baseline, and first evaluation imaging, respectively. $S_{\text {PRE}}$, $\mathrm{S}_{0}, \mathrm{~S}_{\mathrm{POST}}$ stand for the tumor burden (irRECIST) at prebaseline, baseline, and first evaluation imaging, respectively. So the measurable new lesions will be added into the total tumor burden. Besides, if there is no target lesion at pre-baseline, target lesions chosen at baseline will be retrospectively analyzed at pre-baseline. The prebaseline TGK (TGK PRE ) was defined as the difference of the tumor burden per unit of time between pre-baseline and baseline imaging: $\left(\mathrm{S}_{0}-\mathrm{S}_{\mathrm{PRE}}\right) /\left(\mathrm{T}_{0}-\mathrm{T}_{\mathrm{PRE}}\right)$. Similarly, the post-baseline TGK (TGK $\mathrm{TOST})$ was defined as $\left(\mathrm{S}_{\mathrm{POST}^{-}}\right.$ $\left.\mathrm{S}_{0}\right) /\left(\mathrm{T}_{\mathrm{POST}}-\mathrm{T}_{0}\right)$. The TGK ratio (TGKR) was defined as the ratio of TGK $\mathrm{POST}_{\mathrm{P}}$ to $\mathrm{TGK}_{\mathrm{PRE}}$. Hyperprogression was defined as $T G K R \geq 2$.

For the categorical variable data $\chi^{2}$ or Fisher's exact test was used and for the numerical variable data $t$ test or Mann-Whitney test was used. Data input and statistical analysis were performed using SPSS 21.0 statistical software. The significance test was a two-sided test and $P<$ 0.05 considered statistically significant differences.

\section{Results}

We analyzed a total of 45 patients with malignant tumors of digestive system who enrolled and treated in phase I clinical trials with PD-1/PD-L1 inhibitor (alone or combined with CTLA-4 inhibitor) in Peking University Cancer Hospital between August 2016 and May 2017. All of them had the baseline CT scans. As illustrated in the flowchart (Fig. 1), a total of 8 patients (18\%) terminated treatment because of clinical progression or toxicity before the first tumor evaluation. Of the other patients, 12 patients (27\%) did not have a previous CT scan available before baseline. Then 25 patients (56\%) could be explored for TGK $\mathrm{PRE}_{\mathrm{PRE}}$ and TGK POST.

Patient characteristics are described in Table 1. Median age was 54 years. Primary tumor locations were stomach,

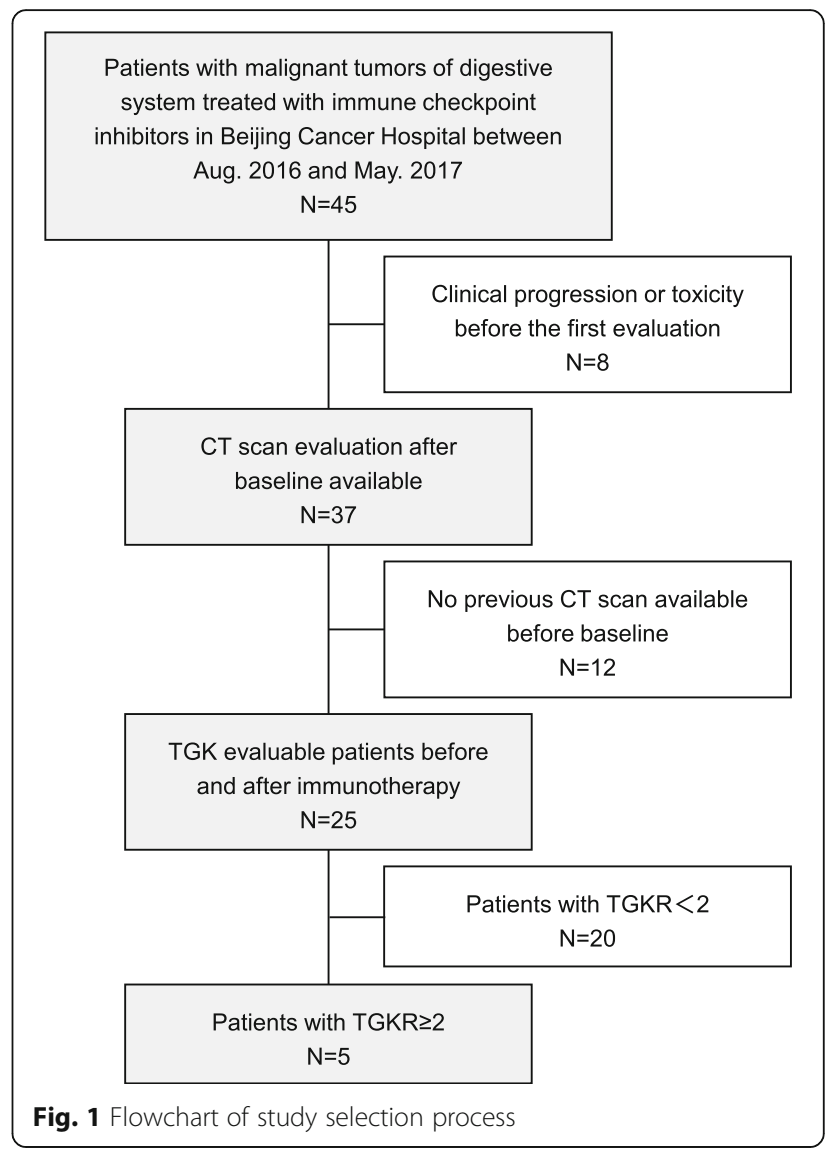

esophagus, colorectal, liver, pancreas and ampulla in 8 (32\%), 7 (28\%), 7 (28\%), 1 (4\%), 1 (4\%) and 1 (4\%) patients, respectively.

By irRECIST, a total of $15(60 \%), 8(32 \%)$ and $2(8 \%)$ patients exhibited progressive disease (PD), stable disease (SD) and partial response (PR), respectively. The distribution of TGK on immunotherapy and TGK preimmunotherapy are shown in Fig. 2. Patients with $\mathrm{TGK}_{\mathrm{POST}}>0$ meant that tumor growth and $\mathrm{TGK}_{\mathrm{POST}}<0$ meant tumor shrinkage. And the slope connect the dot and original point indicated the tumor growth rate, $\mathrm{TGK}_{\mathrm{POST}} / \mathrm{TGK}_{\mathrm{PRE}}>1$ meant tumor growth acceleration and $\mathrm{TGK}_{\mathrm{POST}} / \mathrm{TGK}_{\mathrm{PRE}}<1$ meant tumor growth deceleration. Hyperprogression was observed in 5 patients. Three of 5 were neuroendocrine carcinomas (NECs) and the other 2 were adenocarcinomas. Four of 5 were treated with PD-L1 inhibitor, the other one was treated with PD-L1 inhibitor combined with CTLA-4 inhibitor.

Pseudoprogression was observed in 2 patients with colon carcinomas. The first evaluation after immunotherapy showed PD with TGKR of 1.67 and 0.11 respectively but the general condition was improved which encouraged continued immunotherapy. The second evaluation of the 2 patients both showed SD which confirmed the pseudoprogression. 
Table 1 Patient characteristics

\begin{tabular}{|c|c|c|c|c|}
\hline & All patients $(n=25)$ & TGKR<2 $(n=20)$ & $\operatorname{TGKR} \geq 2(n=5)$ & $P$ value \\
\hline \multicolumn{5}{|l|}{ Gender } \\
\hline Male & $17(68 \%)$ & $14(70 \%)$ & $3(60 \%)$ & \multirow[t]{2}{*}{1.000} \\
\hline Female & $8(32 \%)$ & $6(30 \%)$ & $2(40 \%)$ & \\
\hline Age & $54(22-77)$ & $52(22-77)$ & $63(31-65)$ & 0.587 \\
\hline \multicolumn{5}{|l|}{ EGOG } \\
\hline 0 & $14(56 \%)$ & $10(50 \%)$ & $4(80 \%)$ & \multirow[t]{2}{*}{0.341} \\
\hline 1 & $11(44 \%)$ & $10(50 \%)$ & $1(20 \%)$ & \\
\hline \multicolumn{5}{|l|}{ Location } \\
\hline stomach & $8(32 \%)$ & $6(30 \%)$ & $2(40 \%)$ & \multirow[t]{6}{*}{1.000} \\
\hline esophagus & $7(28 \%)$ & $6(30 \%)$ & $1(20 \%)$ & \\
\hline colorectal & $7(28 \%)$ & $5(25 \%)$ & $2(40 \%)$ & \\
\hline liver & $1(4 \%)$ & $1(5 \%)$ & 0 & \\
\hline ancreas & $1(4 \%)$ & $1(5 \%)$ & 0 & \\
\hline ampulla & $1(4 \%)$ & $1(5 \%)$ & 0 & \\
\hline \multicolumn{5}{|l|}{ Histology } \\
\hline adenocarcinoma & $14(56 \%)$ & $12(60 \%)$ & $2(40 \%)$ & \multirow[t]{4}{*}{0.032} \\
\hline squamous carcinoma & $6(24 \%)$ & $6(30 \%)$ & 0 & \\
\hline neuroendocrine carcinoma & $4(16 \%)$ & $1(5 \%)$ & $3(60 \%)$ & \\
\hline hepatocellular carcinoma & $1(4 \%)$ & $1(5 \%)$ & 0 & \\
\hline \multicolumn{5}{|l|}{ Metastatic site } \\
\hline$\leq 2$ & $16(64 \%)$ & $13(65 \%)$ & $3(60 \%)$ & \multirow[t]{2}{*}{0.749} \\
\hline$>2$ & $9(36 \%)$ & $7(35 \%)$ & $2(40 \%)$ & \\
\hline \multicolumn{5}{|l|}{ Type of immunotherapy } \\
\hline PD-1 inhibitor & $6(24 \%)$ & $6(30 \%)$ & 0 & \multirow[t]{3}{*}{0.447} \\
\hline PD-L1 inhibitor & $16(64 \%)$ & $12(60 \%)$ & $4(80 \%)$ & \\
\hline PD-L1 + CTLA-4 inhibitor & $3(12 \%)$ & $2(10 \%)$ & $1(20 \%)$ & \\
\hline \multicolumn{5}{|l|}{ MMR } \\
\hline $\mathrm{pMMR}$ & $6(40 \%)$ & $4(33 \%)$ & $2(66.7 \%)$ & \multirow[t]{2}{*}{0.525} \\
\hline $\mathrm{dMMR}$ & $9(60 \%)$ & $8(67 \%)$ & $1(33.3 \%)$ & \\
\hline \multicolumn{5}{|l|}{ PD-L1 } \\
\hline positive & $6(42 \%)$ & $5(46 \%)$ & $1(50 \%)$ & \multirow[t]{2}{*}{1.000} \\
\hline negative & 7 (54\%) & $6(55 \%)$ & $1(50 \%)$ & \\
\hline
\end{tabular}

PD-L1 positive meant combined positive score $\geq 1 \%$ and PD-L1 negative meant combined positive score $<1 \%$. Abbreviation: MMR mismatch repair, $p M M R$ mismatch repair proficient, $d M M R$ mismatch repair deficient

\section{Case reports}

We describe all 5 patients with malignant tumors of digestive system treated with ICIs who were considered as hyperprogressors. Serial imaging before and after immunotherapy in the five hyperprogressors are shown in Fig. 3 and variation of the tumor burden are shown in Fig. 4.

\section{Case \#1}

A 31-year-old woman with right colon signet-ring cell carcinoma metastatic to the peritoneum was started on the PD-L1 inhibitor atezolizumab. Immunohistochemistry showed that microsatellite instability-high (MSI-H) and PD-L1 (-). Prior therapies included radical operation for right colon carcinoma followed by S1/oxaliplatin. Restaging imaging done 1.2 months after starting atezolizumab showed multiple new metastases including right breast, bilateral ovaries, T3, T9, T12, L2 and lymphadenopathy (863\% increase from baseline imaging) (Fig. 3a). Superficial lymph node biopsy at the time of progression did not reveal signs of pseudoprogression, including lymphocyte infiltration or tumor necrosis. Patient's performance status fell sharply and died 3.6 months from starting atezolizumab. 


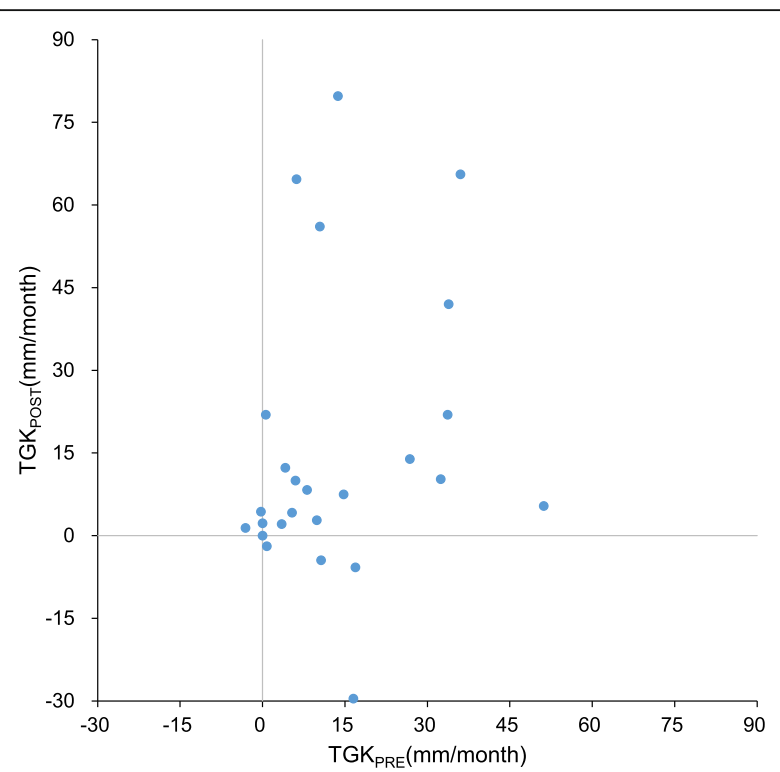

Fig. 2 Pairwise comparisons of TGK on immunotherapy (TGK and TGK pre-immunotherapy (TGK PRE) in 25 patients with malignant tumors of digestive system enrolled and treated in phase I clinical trials with ICls. Each dot represents a patient

\section{Case \#2}

A 63-year-old woman with Her-2 positive gastric adenocarcinoma showed slow progressive liver, lung and peritoneum metastases while sequentially received trastuzumab/ capetacibine/oxaliplatin, paclitaxel/capetacibine, and pyrithione et al. The first evaluation done 1.4 months after the initiation of atezolizumab revealed a rapid progression of liver masses as well as new liver metastasis (107\% increase from baseline imaging) (Fig. 3b). Liver mass biopsy afterwards excluded the possibility of pseudoprogression. Patient subsequently received liver interventional therapy and died 7.4 months from the initiation of atezolizumab.

\section{Case \#3}

A 63-year-old man with colon NEC metastatic to lung, liver, spleen, peritoneum and lymph nodes had palliative surgery followed by first-line therapy with etoposide/cisplatin. Afterwards liver interventional therapy was done but the efficacy was limited. After two cycle's atezolizumab therapy, patient presented severe abdominal distension, which prompted the physician to obtain CT imaging. Scans $(0.94$ months post atezolizumab) showed rapid progression of the peritoneum and liver metastases as well as new brain and adrenal gland metastases $(139 \%$ increase from baseline imaging) (Fig. 3c). Patient received radiotherapy to the brain metastases but died 2.1 months from starting atezolizumab.

\section{Case \#4}

A 65-year-old man with gastric NEC metastatic to liver received radical operation for gastric cancer and adjuvant therapy with etoposide and cisplatin. Therapy was switched to capecitabine and irinotecan after PD. Surveillance imaging demonstrated increasing liver masses and therapy was changed to atezolizumab. CT scans (1.4 months post atezolizumab) revealed a $44 \%$ increase in the liver mass (Fig. 3d) and patient died 5.6 months from the initiation of atezolizumab.

\section{Case \#5}

A 49-year-old man with esophagus NEC metastatic to mediastinal lymph nodes received chemotherapy with etoposide/cisplatin and then concurrent chemoradiotherapy. Therapy was changed to PD-L1 inhibitor durvalumab and CTLA-4 inhibitor tremelimumab after PD. One month later he had an incomplete bowel obstruction which prompted the physician to obtain imaging beforehand. Scans (1.2 months post durvalumab and tremelimumab) showed new lung, liver, T10-12 and L2-4 metastases (538\% increase from baseline imaging) (Fig. 3e) which resulted in backache and paralysis of lower limbs. He died 3.8 months from starting durvalumab and tremelimumab.

\section{Discussion}

As hyperprogression has been reported for a limited time, there is no uniform definition of hyperprogression as mentioned above. Firstly, the evaluation criteria for hyperprogression are different. Nowadays RECIST 1.1 [11] is widely applied in solid tumor evaluation. However, limitations exist when considering immunotherapy. ICIs could impact host antitumor response and may require additional time to achieve measurable or sustained clinical effects compared with traditional cytotoxic chemotherapy [12]. Immune-related response patterns have been observed in clinical trials, including increased size of tumor lesions or development of new lesions which are inflammatory cell infiltrates of immune cells or necrosis with subsequent decreased tumor burden [6,7]. These pseudoprogression would have been classified prematurely as PD by WHO or RECIST 1.1 criteria. So the immune-related response criteria including irRC [7] and irRECIST [13] were published in 2009 and 2014. The core novelty of the irRC and irRECIST is the incorporation of measurable new lesions into total tumor burden and comparison of this variable to baseline measurements [12].

In addition, the evaluation criteria mentioned above are based on changes in tumor size at two time points but not take into account tumor growth dynamics. Fast-growing tumors are more likely to be classified as SD or PD even if the therapy has an antitumor activity, meanwhile slowgrowing tumors are likely to be classified as SD even if there is none antitumor activity [14]. The researchers used different indicators including TGR, TGK and PP to evaluate tumor growth dynamics and the specific formulas are shown in Table 2. There is one question that if there is no 


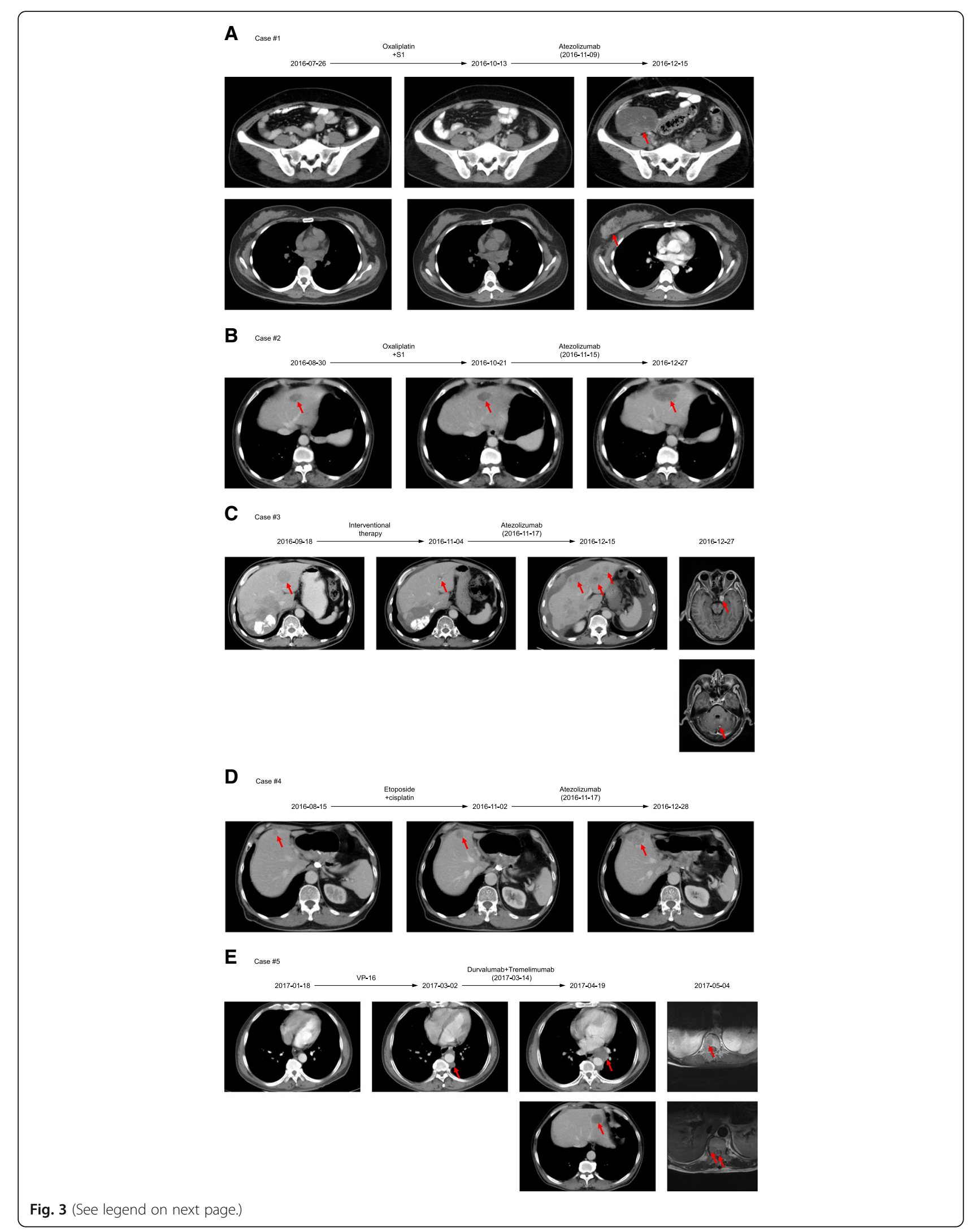


(See figure on previous page.)

Fig. 3 Serial imaging before and after immunotherapy in the five hyperprogressors. Pre-baseline imaging refers to images about 2 months before immunotherapy. Baseline imaging refers to imaging immediately before immunotherapy. a, Case \#1: patient with right colon signet-ring cell carcinoma. Restaging imaging done 1.2 months after starting atezolizumab showed multiple new metastases including right breast, bilateral ovaries et al. (863\% increase from baseline imaging). Patient died 3.6 months from starting atezolizumab. b. Case \#2: patient with gastric adenocarcinoma. The first evaluation done 1.4 months after the initiation of atezolizumab revealed a rapid progression of liver masses as well as new liver metastasis (107\% increase from baseline imaging). Patient subsequently received liver interventional therapy and died 7.4 months from the initiation of atezolizumab. c, Case \#3: patient with colon NEC. After two cycle's atezolizumab therapy, patient presented severe abdominal distension, scans (0.94 months post atezolizumab) showed rapid progression of the peritoneum and liver metastases and new brain and adrenal gland metastases (139\% increase from baseline imaging). Patient died 2.1 months from starting atezolizumab. d, Case \#4: patient with gastric NEC. CT scans (1.4 months post atezolizumab) revealed a 44\% increase in the liver mass and died 5.6 months from the initiation of atezolizumab. $\mathbf{e}$ Case \#5: patient with esophagus NEC. Patient had an incomplete bowel obstruction after immunotherapy and scans (1.2 months post durvalumab and tremelimumab) showed new lung, liver, T10-12 and L2-4 metastases (538\% increase from baseline imaging). He died 3.8 months from starting durvalumab and tremelimumab

measurable lesion before immunotherapy, TGR and PP would be meaningless and cannot be calculated. If these patients have a significant increased size of tumor lesions or new lesions after immunotherapy, they should be highly suspected as hyperprogression and then only the TGK could applicable.

All above we think irRC and irRECIST should be applied when evaluating immunotherapy efficacy. In immunerelated PD (irPD) patients, whether they have clinical benefits should be considered to inform the possibility of pseudoprogression which would decide the subsequent therapy. In the real irPD patients, tumor growth dynamics indicators mentioned above should be used to confirm patients who have hyperprogression. Researchers generally take the indicators as a $\geq 2$-flod increase compare with preimmunotherapy as the definition of hyperprogression. However there is no uniform threshold so far and large sample of research is necessary to determine the appropriate threshold.
Although ICIs have demonstrated salutary antitumor effects, including long-term remissions, it cannot be denied immunotherapy will aggravate the condition in some patients. It is necessary to identify predictors of hyperprogression in order not to treat these patients who might be harmed by ICIs. There are now several biomarkers partially capable of predicting response: PD-L1 expression/amplification, high tumor mutational burden and mismatch repair gene defects $[2,4,15-19]$. However there is no explicit evidence whether these biomarkers could predict the occurrence of hyperprogression. In our study, 15 patients had the detection result of MMR and 13 patients had that of PD$\mathrm{L} 1$, in these patients there is no significant relationship between hyperprogression with MMR or PD-L1 status.

Champiat et al. [8] found that hyperprogression was closely related to age, the median age of hyperprogression and non-hyperprogression patients was 66 and 55 years old respectively $(P=0.007)$. In elderly patients $(\geq 65$ years old $)$ the incidence of hyperprogression was $19 \%$ while $<5 \%$ in

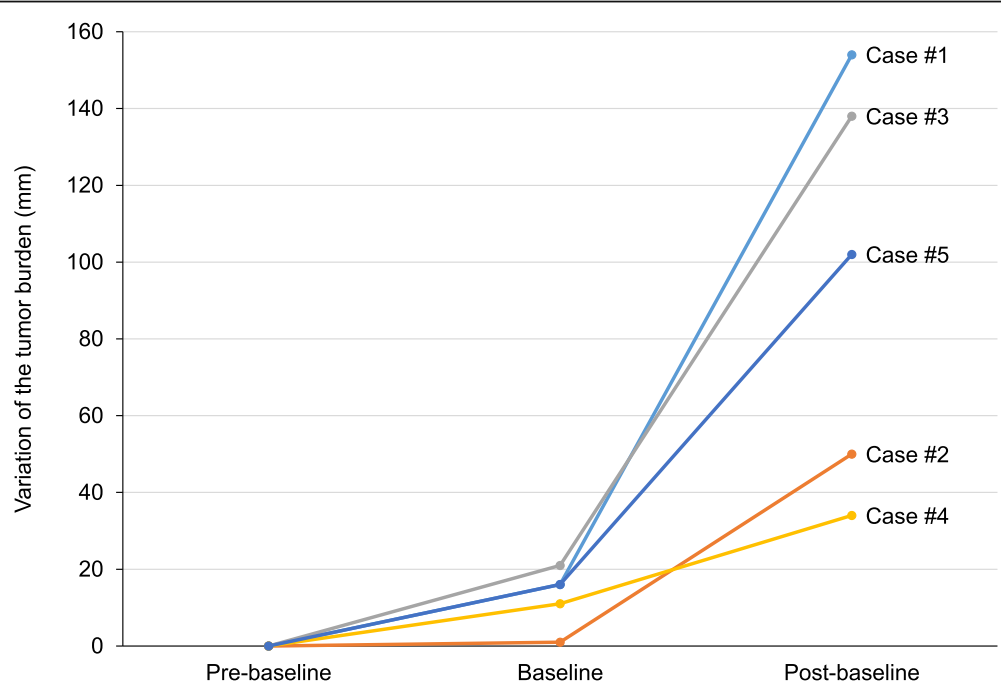

Fig. 4 Variation of the tumor burden in the five hyperprogressors. Tumor burden is compared from about 2 months before immunotherapy (prebaseline) to image immediately before immunotherapy (baseline), and then to first imaging after immunotherapy (post-baseline). Tumor burden was evaluated with irRECIST 
Table 2 The related parameters of hyperprogression according to different researchers

\begin{tabular}{|c|c|c|c|}
\hline & Champiat et al. [8] & Kato et al. [9] & Saada-Bouzid et al. [10] \\
\hline Evaluation criteria & RECIST 1.1 & irRC & $\begin{array}{l}\text { RECIST } 1.1 \\
\text { irRECIST }\end{array}$ \\
\hline $\begin{array}{l}\text { Tumor growth } \\
\text { dynamics indicator }\end{array}$ & TGR & PP & TGK \\
\hline Specific formula & $\begin{array}{l}\mathrm{TG}=3 \mathrm{Log}\left(\mathrm{S}_{\mathrm{T}} / \mathrm{S}_{0}\right) /\left(\mathrm{T}-\mathrm{T}_{0}\right) \\
\mathrm{TGR}=100(\mathrm{TG}-1)\end{array}$ & $P P=\left(S_{T}-S_{0}\right) / S_{0}$ & $\mathrm{TGK}=\left(\mathrm{S}_{\mathrm{T}}-\mathrm{S}_{0}\right) /\left(\mathrm{T}-\mathrm{T}_{0}\right)$ \\
\hline Definition of hyperprogression & PD TGRPOST $/$ TGRPRE $\geq 2$ & $\begin{array}{l}\text { TTF }<2 \text { months } \\
\text { SPOST }_{\text {P }} \text { PRE } \geq 150 \% \\
\text { PP }_{\text {POST }} / \mathrm{PP}_{\text {PRE }}>2\end{array}$ & $\mathrm{TGK}_{\mathrm{POST}} / \mathrm{TGK}$ PRE $\geq 2$ \\
\hline
\end{tabular}

$T$ and $T_{0}$ stand for two time points respectively. $S_{T}$ and $S_{0}$ stand for the sum of tumor burden at $T$ and $T_{0}$ respectively. TGR $R_{P R E}$ Stands for the TGR calculated between pre-baseline and baseline, TGR POST stands for the TGR calculated between baseline and first evaluation imaging. And the other subscripts of "PRE" and "POST" have the similar meanings. Abbreviation: TGR, tumor growth rate; PP, progression pace; TGK, tumor growth kinetics; PD, progressed disease; TTF, time to treatment failure

$<65$ years old patients $(P=0.018)$. In our study the same tendency appeared, the median age in hyperprogressors was 63 and that in non-hyperprogressors was 52 although there was no statistic difference. In elderly patients the function of immune cells, chemotaxis, phagocytosis and intracellular killing of pathogens would decrease [20], but the mechanism associating with hyperprogression is not clear. Saada-Bouzid et al. [10] found that in patients with $\mathrm{R} / \mathrm{M}$ HNSCC hyperprogression significantly correlated with the presence of a regional recurrence $(90 \%$ versus $37 \%, P=0.008$ ). Kato et al. [9] investigated potential genomic markers associated with hyperprogression after immunotherapy and the results showed that MDM2/ MDM4 and EGFR alterations were correlated with TTF < 2 months $(P=0.001, P=0.004)$. Four of 6 patients with MDM2/MDM4 amplification and 2 of 10 patients EGFR aberration had hyperprogression. Further research found that patients with hyperprogression were all treated with PD-1/PD-L1 inhibitor, not with CTLA-4 inhibitor. ICIs could elevate the level of interferon (IFN)- $\gamma$ [21], which in turn activates JAK-STAT signaling [22] leading to an increase in interferon regulatory factor (IRF)-8 expression [23]. Then IRF- 8 binds to the MDM2/MDM4 promoter inducing their expression [23, 24], which could inhibits the p53 tumor suppressor $[25,26]$. And when in the presence of MDM2/MDM4 amplification, hyperprogression could occur [9]. All above is a hypothesis and the exact mechanism linking MDM2/MDM4 amplification and hyperprogression is unclear.

The phenomenon of hyperprogression suggests that in some patients ICIs may promote tumor proliferation instead of repressing growth. It has been confirmed that cell-intrinsic PD-1 receptor could lead to tumor growth in melanoma [27]. And the immune system could promote tumor cells progression and metastasis by inducing local inflammation, DNA damage, angiogenesis, and matrix degradation et al. [28-30]. In addition, ICIs may also result in the upregulation of alternative immune checkpoints [31], and the overall effect is uncertain. ICIs are very likely to promote tumor proliferation via regulating the immune system.

In this study, we explored hyperprogression after immunotherapy in patients with malignant tumors of digestive system. TGK was used to evaluate tumor growth dynamics and hyperprogression was observed in $20 \%$ (5/ 25) of evaluable patients or $11.1 \%(5 / 45)$ of all patients. Three of 5 were NECs and the other 2 were adenocarcinomas. As we all know, NECs in digestive system are a group of highly malignant neoplasms. Patients live a median of 4-15.6 months after their diagnosis [32] and without treatment survival is merely 1 month [33]. The histopathology of 3 patients were all poorly differentiated carcinomas with Ki-67 index of 25-50, 50 and 90\%, respectively. The NECs grow with a high proliferation index, but the grow rate is further increased after immunotherapy. Whether patients with NECs are likely to have hyperprogression is uncertain due to the small size of our series.

Our study had its limitations as well. The number of evaluated patients was small which limited the identification of clinicopathological features of hyperprogression. We elaborated the phenomenon of hyperprogression after immunotherapy in patients with malignant tumors of digestive system preliminarily. And with clinical trials launching and ICIs coming into the market, more patients would have the opportunity to receive immunotherapy, we are largening the sample of patients. We analyzed the rate of change of tumor burden with CT scans and evaluation time in this study, furthermore radiomics analyze would be our next research direction.

\section{Conclusions}

In summary, our study demonstrated that hyperprogression was observed in a fraction of patients with malignant tumors of digestive system treated with ICIs. The definition and predictors of hyperprogression have not evaluated accurately, further research involving more patients treated with ICIs are needed. 


\section{Abbreviations}

CTLA-4: Cytotoxic T lymphocyte associated antigen-4; ICls: Immune checkpoint inhibitors; IRF: Interferon regulatory factor; irPD: immune-related PD; MSI-H: Microsatellite instability-high; NECs: Neuroendocrine carcinomas; PD-1: Programmed cell death-1; PD-L1: Programmed cell death ligand 1; PP: Progression pace; R/M HNSCC: Recurrent and/or metastatic head and neck squamous cell carcinoma; TGK: Tumor growth kinetics; TGKR: Tumor growth kinetics ratio; TGR: Tumor growth rate; TTF: Time to treatment failure

\section{Acknowledgements}

Not applicable.

\section{Authors' contributions}

ZJ was involved in acquisition of data, analysis and interpretation of data and drafting of the manuscript. ZP was involved in acquisition of data, analysis and interpretation of data, drafting of the manuscript and critical revision of the manuscript for important intellectual content. JFG was involved in acquisition of data, analysis and interpretation of data and critical revision of the manuscript for important intellectual content. XTZ was involved in acquisition of data, analysis and interpretation of data, critical revision of the manuscript for important intellectual content. JL was involved in acquisition of data, analysis and interpretation of data, critical revision of the manuscript for important intellectual content. ML was involved in acquisition of data, analysis and interpretation of data, critical revision of the manuscript for important intellectual content. ZHL was involved in acquisition of data, analysis and interpretation of data, critical revision of the manuscript for important intellectual content. LS was involved in study concept and design, analysis and interpretation of data, critical revision of the manuscript for important intellectual content and study overall supervision. All authors were involved in critically revising the manuscript prior to final submission. All authors read and approved the final manuscript.

\section{Funding}

This study was supported by the National Key Research and Development Program of China (No. 2017YFC1308900) (data collection and analysis), Beijing Municipal Administration of Hospitals' Youth Program (QML20171102) (data collection and analysis), Clinical Medicine Plus X-Young Scholars Project of Peking University (interpretation of data).

\section{Availability of data and materials}

The datasets used and/or analyzed during the current study are available from the corresponding author on reasonable request.

\section{Ethics approval and consent to participate}

This study was approved by the Beijing Cancer Hospital Ethics Committee. Written consent was obtained from all individual participants included in this study. All procedures performed in studies involving human participants were in accordance with the ethical standards of the institutional and/or national research committee and with the 1964 Helsinki declaration and its later amendments or comparable ethical standards. The institutional review board at our hospital approved this study.

\section{Consent for publication}

Written consent for publication was obtained from all individual participants included in this study. All participants consented to the publication of potentially identifying information and images in the study. And this consent was obtained in writing form from participants.

\section{Competing interests}

The authors including Lin Shen and Xiaotian Zhang are members of the editorial board of this journal. And no other competing interests were declared.

Received: 21 March 2019 Accepted: 10 July 2019

Published online: 17 July 2019

\section{References}

1. Robert C, Long GV, Brady B, Dutriaux C, Maio M, Mortier L, Hassel JC, Rutkowski $P$, McNeil C, Kalinka-Warzocha E, et al. Nivolumab in previously untreated melanoma without BRAF mutation. N Engl J Med. 2015;372(4):320-30.
2. Le DT, Uram JN, Wang $H$, Bartlett $B R$, Kemberling $H$, Eyring $A D$, Skora $A D$, Luber BS, Azad NS, Laheru D, et al. PD-1 blockade in tumors with mismatchrepair deficiency. N Engl J Med. 2015;372(26):2509-20.

3. Brahmer J, Reckamp KL, Baas P, Crino L, Eberhardt WE, Poddubskaya E, Antonia S, Pluzanski A, Vokes EE, Holgado E, et al. Nivolumab versus docetaxel in advanced squamous-cell non-small-cell lung Cancer. N Engl J Med. 2015;373(2):123-35.

4. Borghaei H, Paz-Ares L, Horn L, Spigel DR, Steins M, Ready NE, Chow LQ, Vokes EE, Felip E, Holgado E, et al. Nivolumab versus docetaxel in advanced nonsquamous non-small-cell lung Cancer. N Engl J Med. 2015;373(17):1627-39.

5. Muro K, Chung HC, Shankaran V, Geva R, Catenacci D, Gupta S, Eder JP, Golan T, Le DT, Burtness B, et al. Pembrolizumab for patients with PD-L1positive advanced gastric cancer (KEYNOTE-012): a multicentre, open-label, phase 1b trial. The Lancet Oncology. 2016;17(6):717-26.

6. Di Giacomo AM, Danielli R, Guidoboni M, Calabro L, Carlucci D, Miracco C, Volterrani L, Mazzei MA, Biagioli M, Altomonte M, et al. Therapeutic efficacy of ipilimumab, an anti-CTLA-4 monoclonal antibody, in patients with metastatic melanoma unresponsive to prior systemic treatments: clinical and immunological evidence from three patient cases. Cancer Immunol Immunother. 2009;58(8):1297-306.

7. Wolchok JD, Hoos A, O'Day S, Weber JS, Hamid O, Lebbe C, Maio M, Binder M, Bohnsack O, Nichol G, et al. Guidelines for the evaluation of immune therapy activity in solid tumors: immune-related response criteria. Clin Cancer Res. 2009;15(23):7412-20.

8. Champiat S, Dercle L, Ammari S, Massard C, Hollebecque A, Postel-Vinay S, Chaput N, Eggermont A, Marabelle A, Soria J-C, et al. Hyperprogressive disease is a new pattern of progression in Cancer patients treated by antiPD-1/PD-L1. Clin Cancer Res. 2017;23(8):1920-8.

9. Kato S, Goodman AM, Walavalkar V, Barkauskas DA, Sharabi A, Kurzrock R. Hyperprogressors after immunotherapy:analysis of genomic alterations associated with accelerated growth rate. Clin Cancer Res. 2017;23(15):4242-50.

10. Saada-Bouzid E, Defaucheux C, Karabajakian A, Palomar Coloma V, Servois V, Paoletti X, Even C, Fayette J, Guigay J, Loirat D, et al. Hyperprogression during anti-PD-1/PD-L1 therapy in patients with recurrent and/or metastatic head and neck squamous cell carcinoma. Ann Oncol. 2017;28:1605-11.

11. Eisenhauer EA, Therasse P, Bogaerts J, Schwartz LH, Sargent D, Ford R, Dancey J, Arbuck S, Gwyther S, Mooney M, et al. New response evaluation criteria in solid tumours: revised RECIST guideline (version 1.1). Eur I Cancer. 2009;45(2):228-47.

12. Chiou VL, Burotto M. Pseudoprogression and immune-related response in solid tumors. J Clin Oncol. 2015;33(31):3541-3.

13. Bohnsack $\mathrm{O}$, Hoos A, Ludajic K. Adaptation of the immune-related response criteria: irRECIST. Ann Oncol. 2014;25(suppl 4):iv361.

14. Ferte C, Fernandez M, Hollebecque A, Koscielny S, Levy A, Massard C, Balheda R, Bot B, Gomez-Roca C, Dromain C, et al. Tumor growth rate is an early Indicator of antitumor drug activity in phase I clinical trials. Clin Cancer Res. 2013;20(1):246-52

15. Patel SP, Kurzrock R. PD-L1 expression as a predictive biomarker in Cancer immunotherapy. Mol Cancer Ther. 2015;14(4):847-56.

16. Llosa NJ, Cruise M, Tam A, Wicks EC, Hechenbleikner EM, Taube JM, Blosser $\mathrm{RL}$, Fan $\mathrm{H}$, Wang $\mathrm{H}$, Luber BS, et al. The vigorous immune microenvironment of microsatellite instable colon cancer is balanced by multiple counter-inhibitory checkpoints. Cancer Discov. 2015;5(1):43-51.

17. Boland CR, Goel A. Microsatellite instability in colorectal cancer. Gastroenterology. 2010;138(6):2073-87.

18. Campesato LF, Barrososousa R, Jimenez L, Correa BR, Sabbaga J, Hoff PM, Reis LF, Galante PA, Camargo AA. Comprehensive cancer-gene panels can be used to estimate mutational load and predict clinical benefit to PD-1 blockade in clinical practice. Oncotarget. 2015;6(33):34221-7.

19. Hugo W, Zaretsky JM, Sun L, Song C, Moreno BH, Hulieskovan S, Berentmaoz B, Pang J, Chmielowski B, Cherry G. Genomic and transcriptomic features of response to anti-PD-1 therapy in metastatic melanoma. Cell. 2016;165(1):35.

20. Solana R, Tarazona R, Gayoso I, Lesur O, Dupuis G, Fulop T. Innate immunosenescence: effect of aging on cells and receptors of the innate immune system in humans. Semin Immunol. 2012;24(5):331-41.

21. Peng W, Liu C, Xu C, Lou Y, Chen J, Yang Y, Yagita H, Overwijk WW, Lizée G, Radvanyi L. PD-1 blockade enhances T-cell migration to tumors by elevating IFN- $\gamma$ inducible chemokines. Cancer Res. 2012;72(20):5209-18.

22. Schindler C, Levy DE, Decker T. JAK-STAT signaling: from interferons to cytokines. J Biol Chem. 2007;282(28):20059. 
23. Waight JD, Netherby C, Hensen ML, Miller A, Hu Q, Liu S, Bogner PN, Farren MR, Lee KP, Liu K. Myeloid-derived suppressor cell development is regulated by a STAT/RF-8 axis. J Clin Investig. 2013;123(10):4464.

24. Zhou JX, Chang HL, Chen FQ, Wang H, Naghashfar Z, Abbasi S, Morse HC. IFN regulatory factor 8 regulates MDM2 in germinal center $B$ cells. J Immunol. 2009;183(5):3188-94.

25. Zhao Y, Yu H, Hu W. The regulation of MDM2 oncogene and its impact on human cancers. Acta Biochim Biophys Sin. 2014;46(3):180.

26. Wade M, Li YC, Wahl GM. MDM2, MDMX and p53 in oncogenesis and cancer therapy. Nat Rev Cancer. 2013;13(2):83.

27. Kleffel S, Posch C, Barthel SR, Mueller H, Schlapbach C, Guenova E, Elco CP, Lee N, Juneja VR, Zhan Q, et al. Melanoma cell-intrinsic PD-1 receptor functions promote tumor growth. Cell. 2015;162(6):1242-56.

28. Colotta F, Allavena P, Sica A, Garlanda C, Mantovani A. Cancer-related inflammation, the seventh hallmark of cancer: links to genetic instability. Carcinogenesis. 2009;30(7):1073-81.

29. Pollard JW. Tumour-educated macrophages promote tumour progression and metastasis. Nat Rev Cancer. 2004;4(1):71-8.

30. Guo X, Zhai L, Xue R, Shi J, Zeng Q, Gao C. Mast cell Tryptase contributes to pancreatic Cancer growth through promoting angiogenesis via activation of Angiopoietin-1. Int J Mol Sci. 2016;17(6):834.

31. Koyama S, Akbay EA, Li YY, Herter-Sprie GS, Buczkowski KA, Richards WG, Gandhi L, Redig AJ, Rodig SJ, Asahina H, et al. Adaptive resistance to therapeutic PD-1 blockade is associated with upregulation of alternative immune checkpoints. Nat Commun. 2016;7:10501.

32. Ilett EE, Langer SW, Olsen IH, Federspiel B, Kjaer A, Knigge U. Neuroendocrine carcinomas of the Gastroenteropancreatic system: a comprehensive review. Diagnostics (Basel). 2015;5(2):119-76.

33. Sorbye H, Welin S, Langer SW, Vestermark LW, Holt N, Osterlund P, Dueland S, Hofsli E, Guren MG, Ohrling K, et al. Predictive and prognostic factors for treatment and survival in 305 patients with advanced gastrointestinal neuroendocrine carcinoma (WHO G3): the NORDIC NEC study. Ann Oncol. 2013:24(1):152-60

\section{Publisher's Note}

Springer Nature remains neutral with regard to jurisdictional claims in published maps and institutional affiliations.

Ready to submit your research? Choose BMC and benefit from:

- fast, convenient online submission

- thorough peer review by experienced researchers in your field

- rapid publication on acceptance

- support for research data, including large and complex data types

- gold Open Access which fosters wider collaboration and increased citations

- maximum visibility for your research: over $100 \mathrm{M}$ website views per year

At $\mathrm{BMC}$, research is always in progress.

Learn more biomedcentral.com/submissions 